ISSN 1112-9867

http://www.jfas.info

\title{
URINARY TRACT INFECTION AMONG PREGNANT WOMEN AT BOBO-DIOULASSO: EPIDEMIOLOGICAL AND BACTERIOLOGICAL ASPECTS
}

\author{
E. Taale ${ }^{1 *}$, S. Sanou ${ }^{2}$, I. Sangare ${ }^{3}$, A. D. Abdelkerim ${ }^{4}$, A. Mbatna ${ }^{2}$, C. Sirima ${ }^{2}$ and A. \\ Savadogo ${ }^{1}$
}

${ }^{1}$ Laboratoire de Biochimie et d'Immunologie Appliquée (LABIA), Département de Biochimie et Microbiologie, Unité de Formation et de Recherche en Sciences de la Vie et de la Terre (UFR-SVT), Université Ouaga I Pr Joseph KI-ZERBO, 03BP7021 Ouaga 03, Burkina Faso. ${ }^{2}$ Laboratoire de bactériologie et virilogie du Centre Hospitalier Universitaire Souro Sanou (CHUSS) de Bobo-Dioulasso, 01BP676, Bobo-Dioulasso, Burkina Faso.

${ }^{3}$ Unité de Parasitologie, Centre Hopitalier Universitaire Souro Sanou (CHUSS) de Bobo-Dioulasso, 01BP676, Bobo-Dioulasso, Burkina Faso.

${ }^{4}$ Unité de Formation en Sciences et Techniques, Université Catholique de l'Afrique de l'Ouest/ Unité Universitaire à Bobo-Dioulasso (UCAO/UUB), Bobo-Dioulasso, Burkina Faso.

Received: 13 April 2016 / Accepted: 22 August 2016 / Published online: 01 Septembre 2016

\begin{abstract}
Urinary tract infections are the first infections in pregnant women and can cause serious complications during pregnancy. In order to improve its management in low income country like Burkina Faso, we conducted a prospect cross-sectional study, to describe its epidemiological and biological aspects in pregnant women at Souro Sanou hospital at Bobo-Dioulasso (Burkina Faso).
\end{abstract}

Author Correspondence, e-mail: taaleernest12@ hotmail.com doi: $\underline{\text { http://dx.doi.org/10.4314/jfas.v8i3.26 }}$ 
Epidemiologically, the frequency of urinary tract infection was $27.5 \%$ and affected mostly 21 to 30 years pregnant women. Bacteriologically, the main isolates found were: E. coli, Staphylococcus aureus, Staphylococcus epidermis, Serratia, Acinetobacter, Enterobacter and Candida. The 81 isolates showed a good sensitivity to cephalosporin (C3G), aminoglycosides, oxacillin and erythromycin. These antibiotics are recommended for pregnant women before urinary cytobacteriological exam.

Keywords: Antibiotic, Bobo-Dioulasso, E. coli, pregnant woman, urinary tract infection, urinary cytobacteriological exam.

\section{INTRODUCTION}

Selon Thoulon [1], 10 à $20 \%$ des femmes enceintes risques d'être atteintes d'infection urinaire. C'est une infection bénigne dans la majorité des cas car généralement elle est limitée au bas de l'appareil utinaire [2]. L'infection urinaire est la colonisation bactérienne de l'urine et/ou de l'appareil urinaire (structures glandulaires, muqueuses ou parenchymateuses) depuis les reins jusqu'au méat urétral [3]. Elle est souvent primitive c'est-à-dire qu'elle ne s'accompagne pas d'anomalie de l'appareil urinaire [4]. Elle associe le plus souvent une bactériurie à une leucocyturie ; qui peut manquer dans les infections asymptomatiques [5]. Elle est d'une gravité particulière pour la mère et pour le fœtus. Au terme de ces complications on note $[6,7]$ :

- pour la mère : la cystite, la pyélonéphrite, l'insuffisance rénale, l'anémie, la septicémie, la pyonéphrose, la nécrose papillaire, la maladie thromboembolique et voire la mort.

- pour le fotus : l'avortement, l'accouchement prématuré dans $20 \%$ des cas, une infection néonatale, une hypotrophie et la mort périnatale.

Dans les pays du sud, les femmes enceintes négligent l'action des médecins, il s'impose donc de garantir la santé de la femme enceinte et du fœtus. Il existe très peu de données sur les femmes enceintes atteintes d'infection urinaires au Burkina Faso. C'est fort de ce constat que cette étude transversale a été entreprise au Centre Hospitalier Universitaire Souro Sanou (CHUSS) de Bobo-Dioulasso afin de pouvoir apporter quelques données sur cet aspect. De plus les échecs thérapeutiques sont de plus en plus fréquents avec les antibiotiques prescrits 
en premiére intention. Il est donc important de poser un diagnostic étiologique précoce avec l'aide du laboratoire bactériologique afin d'eviter les complications.

La présente étude a donc consisté à déterminer l'etiologie bactérienne des infections urinaires, leur épidémiologie et leur sensibilité aux antibiotiques en vue de contribuer à l'amélioration de la prise en charge de cette pathologie chez la femme enceinte.

\section{MATERIEL ET METHODES}

Cadre de l'étude : le Centre Hospitalier Universitaire Souro Sanou est un hopital de référence à vocation hospitalo-universitaire. Il comporte des services cliniques et des services médico-techniques dont le laboratoire d'analyse médical dans lequel nous avons réalisé notre étude.

Type, population et période d'étude : Nous avons mené une étude transversale prospective à visée descriptive sur une période de trois mois du 15 Février au 15 Mai 2015. Notre étude à porté sur les femmes enceintes présentant des syndromes d'une infection urinaire et dont l'examen cytobactériologique des urines (ECBU) a été démandé.

L'échantillonnage été fait dans trois (3) CSPS à savoir : 1 urbain central (Guimbi Ouattara), 1 urbain périphérique (Bolmakoté) et 1 rural (Yegueresso).

Nous avons effectué un échantillonnage non probabiliste de type accidentel en fonction de l'arrivée des échantillons d'urine au laboratoire et un ensemble de 315 échantillons d'urines a été collecté.

\section{Méthodes d'étude}

Prélevement, transport et conservation des urines : Les prélevements ont été fait avant toute antibiothérapie. En effet, après une toilette minutieuse de la vulve, les urinaires du $1^{\mathrm{er}}$ jet ont été récueillis dans un flacon sterile. Les échantillons d'urines prélevées à l'exterieur du CHUSS ont été achéminés le plus rapidement possible au laboratoire. Lorsque tous les prélevements ne pouvaient être analysés immédiatemment, ils étaient conservé à $+4{ }^{\circ} \mathrm{C}$ au réfrigérateur pendant 6 heures au maximum.

\section{L'examen cytobactériologique des urines}


- Examen direct : Nous avons apprécié l'aspect macroscopique des urines non centrifugées. Puis l'examen des urines à l'état frais avant et après centrifugation pour rechercher les élements figurés tels que les leucocytes, les hématies, les bactéries, les parasites, les levures, les cellules épithéliales, les cylindres et les cristaux.

- La coloration différentielle de Gram a permis de connaître la morphologie des bactéries et leur affinité tinctoriale.

- Examen après culture : les urines ont été ensemencées sur milieux gélosés selectifs et non selectifs (CLED et EMB). L'identification des bacilles à Gram négatifs a été faite sur les colonies suspectes, au moyen de la galerie classique de le Minor ou de la galerie API $20^{\mathrm{E}}$ (BioMarieux, France). Pour les cocci à Gram positifs nous avons utilisés les caractères biochimiques suivants : catalase, culture sur Chapman, test à la Dnase et à la coagulase. L'identification de l'espèce Candida albicans a été faite par le test de filamentation dans de sérum humain après culture sur milieu sabouraud.

Antibiogramme : La sensibilité aux antibiotiques a été réalisé par la méthode classique de diffusion sur milieu gélosé et l'interprétation faite à l'aide de la table des abaques selon la méthode du CASFM 2014. Les antibiotiques utilisés sont: Amoxicilline, Association amoxicilline + acide clavulanique, Céfotaxime, Ciprofloxacine, Céphalexine, Céftriaxone, Gentamicine, Oxacilline, Erythromycine, Céfoxitine et Lincomycine.

Collecte et analyse des données : Pour la collecte des données, nous avons élaboré une fiche de collecte comportant les variables suivantes : âge de la gestante, statut socio-professionnel, terme de la grossesse, parité, germes identifiés et la sensibilité des germes aux antibiotiques. Les données collectées ont été analysées à l'aide du logiciel SPSS version 20.

\section{RESULTATS ET DISCUSSION}

\section{Aspects épidémiologiques}

- Prévalence de l'infection urinaire (IU) : La prévalence de l'IU au CHUSS était de 25,7\%. En 2002, des travaux menés au Burkina Faso par Nikiema [8], ont montré que 18,5\% des femmes enceintes présentaient une infection urinaire. Ainsi on remarque une forte proportion des femmes enceintes infectées à Bobo-Dioullaso, la $2^{\text {ème }}$ ville du pays et capitale 
économique. Des études menées en Tanzanie [9] et au Nigéria [10] ont revelé respectivement que $16,4 \%$ et $23,9 \%$ des femmes enceintes présentaient des infections urinaires. Par contre des taux d'infection inferieurs aux nôtres ont été obtenus par d'autres auteurs lors de leurs études menées respectivement à Bamako [11] et en Guinée [12]: ces taux d'infection sont respectivement de $9,9 \%$ et $8,54 \%$. Cette différence pourrait s'expliquer d'une part par la faible couverture de consultation prénatale et le manque de moyens de dépistage et de traitement des infections urinaires dans nos pays en voie de développement. D'autre part, ce sont des infections souvent asymptomatiques car on note des cas d'infections urinaires qui passent inaperçues.

- Distribution de l'IU selon l'âge : Les femmes enceintes de 21 à 30 ans étaient les plus vulnérables avec un taux de 59,7 \% ; suivies des femmes enceintes de 15 à 20 ans (20,6\%). Diassana [12] a montré que les femmes enceintes de 15 à 20 ans sont les plus vulnérables aux infections urinaires en Guinée. La jeunesse de la population burkinabè pourrait expliquer ce résultat car la moyenne d'âge des femmes enquêtées était de 26,11 $\pm 0,01$ ans.

- Prévalence de l'infection urinaire : sur les 315 échantillons d'urines examinés, une bactérie a été isolée dans 81 cas soit une prévalence de 25,7\%. Donc une bactérie a été retrouvée dans 81 échantillons sur 315 soumis à cette étude.

- Distribution de l'IU selon le terme de la grossesse : $37,10 \%$ des fememes enceintes dont la grossesse est au deuxième trimestre étaient les plus vulnérables, suivies de 36,51\% des femmes enceintes dont la grosseese était au troisième trimestre (Tableau I). Nos résultats sont donc comparables à ceux obtenus par Nikiéma [8] au Burkina Faso car il a mentionné la majorité des infections au deuxième trimestre et une fréquence plus élevée au premier trimestre, a été observée par Olsen et al. [9] en Tanzanie. Dans cette étude le nombre de femmes enceintes dont la grossesse était au $1^{\text {er }}$ trimestre est inférieur à celui des femmes enceintes dont la grossesse était respectivement au $2^{\text {ème }}$ et $3^{\text {ème }}$ trimestre, c'est pourquoi cet écart de résultat s'observe. Si le nombre des femmes enceintes dont la grossesse était au $1^{\text {er }}$ trimestre était égal aux autres effectifs, on pourrait obtenir les mêmes résultats que ceux obtenus par Olsen et al. [9] car au premier trimestre les femmes enceintes négligent souvent les consultations prénatales. Cette négligence peut être due au manque de moyens financiers 
et à certains préjugés sociaux.

Tableau I: Infection urinaire selon l'age de la grossesse

\begin{tabular}{l|lllll}
\hline Age de la grossesse & $\mathbf{1}^{\text {er }}$ Trimestre & $2^{\text {ème }}$ Trimestre & $3^{\text {eme }}$ trimestre & Total \\
\hline Effectif & $83(26,47 \%)$ & $117(37,10 \%)$ & $115(36,51 \%)$ & $315(100 \%)$ & \\
Germes isolés & & & & & \\
Acinetobacter & 2 & 0 & 0 & $2(2,46 \%)$ \\
Candida alibicans & 3 & 5 & 8 & $16(19,75 \%)$ & \\
E. coli & 8 & 13 & 9 & $30(37,03 \%)$ & \\
Enterobacter & 0 & 1 & 0 & $1(1,23 \%)$ & \\
Klebsiella pneumoniae & 0 & 2 & 1 & $3(3,70 \%)$ & $81(100 \%)$ \\
Serratia & 0 & 1 & 0 & $1(1,23 \%)$ & \\
Staphylococcus aureus & 4 & 5 & 9 & $18(22,22 \%)$ & \\
Staphylococcus & 2 & 3 & 5 & $10(12,35 \%)$ & \\
epidermis & & & & & \\
\hline
\end{tabular}

- Distribution de l'IU selon la parité : Les paucipares étaient majoritaires avec 34,29\%, suivie des nullipares avec 28,60\% (Tableau II). Nos résultats sont proches de ceux de Touré [13] au Mali qui avait trouvé 33,3\% de paucipares et 29,2\% de nullipares. Par contre, Nikiéma [8] au Burkina Faso avait obtenu $16 \%$ de paucipares et $35 \%$ de nullipares. Ceci équivaut à dire que l'infection urinaire est fréquente en début de parité.

Tableau II: Répartition des 315 femmes enceintes enquêtées selon la parité

\begin{tabular}{ccc}
\hline & Effectifs & Pourcentage (\%) \\
\hline Nullipare & 90 & 28,60 \\
Primipare & 85 & 26,98 \\
Paucipare & 108 & 34,29 \\
Multipare & 32 & 10,16 \\
Total & $\mathbf{3 1 5}$ & $\mathbf{1 0 0 , 0}$ \\
\hline
\end{tabular}

Nullipare : 0 Enfant ; Primipare : 1 Enfant ; Paucipare : 2 à 3 Enfants et Multipare : 4 à plus

- Distribution de l'IU selon le statut socio-professionnel : Le statut socio-professionnel donne une fréquence plus élevée des ménagères avec 83,49\%, suivie des élèves et étudiantes (Tableau III). Nos résultats sont largement supérieurs à ceux obtenus par Sangare [14] au 
Burkina et Koné [15] au Bénin respectivement de 51,9\% et de 65,7\%. Ceci pourrait être dû au faible taux de scolarisation féminine.

Tableau III: Répartition des femmes selon le statut socio-professionnel

\begin{tabular}{|c|c|c|}
\hline & Effectifs & Pourcentage $(\%)$ \\
\hline Coiffeuse & 6 & 1,91 \\
\hline Commercante & 4 & 1,27 \\
\hline Couturière & 7 & 2,22 \\
\hline Elève/Etudiante & 24 & 7,62 \\
\hline Institutrice & 3 & 0,95 \\
\hline Ménagère & 263 & 83,49 \\
\hline Secrétaire & 4 & 1,27 \\
\hline $\begin{array}{l}\text { Auxiliaire de pharmacie; conseillère sociale; } \\
\text { pompiste et serveuse }\end{array}$ & 4 & 1,27 \\
\hline Total & 315 & 100,0 \\
\hline
\end{tabular}

\section{Aspects bactériologiques}

- Souches bactériennes identifiées : 81 bactéries ont été identifiées dans 81 échantillons sur un total de 315 (Tableau I, Figure 1). Les entérobactéries réprésentent 43,1\% de l'ensemble des bactéries identifiées; les Cocci à Gram positifs (34,5\%), suivi de Candida albicans $(19,75 \%)$ et les Bacilles à Gram négatifs non fermentaires (2,5\%). Nos résultats sont comparables à ceux de Marrakchi et al. [16] à Tunis et Gebre [17] en Ethiopie qui ont trouvé des fréquences plus élevées respectivement de 59,3\% et 62,5\%. Par contre, Olusanya et al. [10] au Nigeria a obtenu une fréquence de $41,2 \%$ pour les entérobactéries. Parmis les entérobactéries, les espèces d' $E$. coli viennent largement en tête avec 37,03\%. Marrakchi et al. [16], Gebre [17] et Olusanya et al. [10] ont trouvés lors de leurs études, les espèces d'E. coli de proportions respectives de 49,1\%; $46 \%$ et 21,3\%. Les espèces de Staphylocoques, Cocci à Gram positifs suivent de près les entérobactéries avec un taux de 34,57 \%. Ce même constat a été fait par Marrakchi et al. [16] et Gebre [17] qui notent également que les cocci à Gram positifs occupaient la $2^{\mathrm{e}}$ place parmis les différentes bactéries isolées chez les femmes enceintes présentant une infection urinaire. Par contre, dans l'étude d'Olusanya et al. [10] au Nigeria les Cocci à Gram positifs viennent en tête avec 55,9\% des germes isolés. Acinetobacter fut le seul genre de Bacille à Gram négatif isolé en dehors des entérobactéries 
$(2,46 \%)$. Il vient en dernière position après les lévures du genre Candida albicans. Dans notre étude, nous avons trouvé 19,75\% de Candida albicans. Contrairement à ceux de Marrakchi et al. [16] et Gebre [17] qui n'ont pas pris en compte les lévures ; alors qu'il est très important de prendre en compte la présence de Candida albicans dans les infections urinaires surtout chez la femme enceinte.

La figure 1 illustre la répartition des germes isolés en fonction de l'âge de la grossesse chez les femmes diagnostiquées.

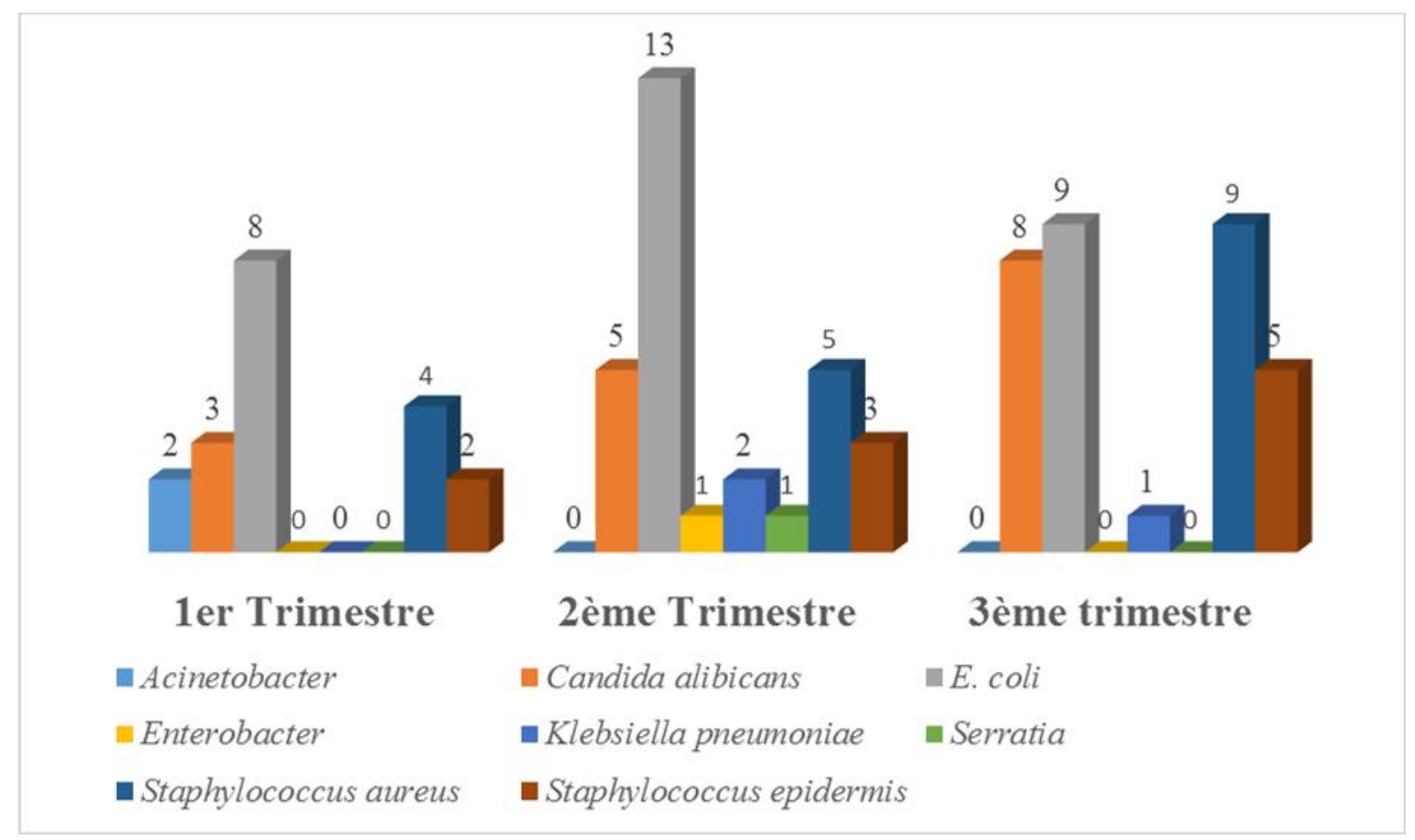

Fig.1. Fréquences des principales espèces bactériennes selon le terme de la grossesse

\section{Sensibilité aux antibiotiques de tous les germes}

Les souches d'E. coli et d'Enterobacter étaient fortement résistantes à l'amoxicilline et à l'association de l'amoxicilline + acide clavulanique (Tableau IV). Par contre, elles présentent une bonne sensibilité aux céphalosporines de troisième génération $(\mathrm{C} 3 \mathrm{G})$, à la céphalexine, à la Céftriaxone, à la gentamicine et à la ciprofloxacine. Ces fortes sensibilités pourraient s'expliquer par la variation de la sensibilité du germe selon l'écosystème.

- Les souches de Klebsiella pneumoniae ont présenté une résistance à l'amoxicilline et à l'amoxicilline + acide clavulanique (100\%) et une bonne sensibilité à la gentamicine, ciprofloxacine et aux C3G (Tableau IV). Nos résultats sont comparables à ceux obtenus par 
Combary [18] au Mali qui avait noté une sensibilité de 90\% aux aminosides. Par contre Seydi et al. [19] à Dakar ont trouvé chez des patientes hospitalisées, un faible taux de sensibilité à la ciprofloxacine $(40 \%)$.

- Les souches de Serratia sont résistantes aux C3G, à la gentamicine, à l'amoxicilline et à l'amoxicilline + acide clavulanique et reste sensible à la ciprofloxacine à 100\%. Nos résultats sont identiques à ceux obtenus par Ouédraogo et al. [20] à Bobo-Dioulasso qui avaient trouvé une résistance à la ciprofloxacine et une sensibilité de 100\% à l'amoxicilline.

- Les souches d'Acinetobacter sont résistantes à $100 \%$ aux pénicillines G et aux C3G (Tableau IV) et présentent une bonne sensibilité à la ciprofloxacine et à la gentamicine (100\%). Nos résultats concordent avec ceux de la littérature dont le genre Acinetobacter est une bactérie fréquemment résistante à de nombreux antibiotiques. C'est le plus souvent une bactérie multi-résistante (BMR).

Tableau IV: Profil de sensibilité globale aux antibiotiques de tous les germes.

\begin{tabular}{|c|c|c|c|c|c|c|}
\hline \multirow[t]{2}{*}{ Antibiotiques } & \multicolumn{6}{|c|}{ Nombre (\%) de souches sensibles } \\
\hline & E. coli & $\begin{array}{l}\text { Klebsiella } \\
\text { pneumoniae }\end{array}$ & Enterobacter & Serratia & Staphylocoque & Acinetobacter \\
\hline Ciprofloxacine & $30(100 \%)$ & $3(100 \%)$ & $1(100 \%)$ & $1(100 \%)$ & $21(75 \%)$ & $2(100 \%)$ \\
\hline Amoxicicilline & $2(6,25 \%)$ & - & & - & $4(14,29 \%)$ & - \\
\hline Gentamicine & $30(100 \%)$ & $3(100 \%)$ & $1(100 \%)$ & - & $21(75 \%)$ & $2(100 \%)$ \\
\hline Erythromycine & - & - & - & - & $17(60,71 \%)$ & - \\
\hline Céfoxitine & - & - & - & - & $11(39,29 \%)$ & - \\
\hline Lincomycine & - & - & - & - & $9(32,14 \%)$ & - \\
\hline $\begin{array}{l}\text { Amoxicilline }+ \\
\text { acide } \\
\text { clavulanique }\end{array}$ & $4(13,33 \%)$ & - & $1(100 \%)$ & - & $4(14,29 \%)$ & - \\
\hline Oxacilline & - & - & $1(100 \%)$ & - & $15(53,57 \%)$ & - \\
\hline Céphalexine & $22(73,33 \%)$ & $3(100 \%)$ & - & - & - & - \\
\hline Céftriaxone & $28(93,33 \%)$ & $2(66,6 \%)$ & - & - & - & - \\
\hline Céfotaxime & $18(60 \%)$ & $3(100 \%)$ & - & - & - & - \\
\hline $\begin{array}{l}\text { Céphalosporines } \\
\text { de troisième } \\
\text { génération }\end{array}$ & $27(90 \%)$ & $3(100 \%)$ & - & - & - & - \\
\hline
\end{tabular}

- : Souches résistantes

- Les souches de Staphylocoque présentent une bonne sensibilité à l'oxacilline, à la 
ciprofloxacine, à la gentamicine et à l'Erythromycine. Certaines souches ont montré une très forte résistance à la pénicilline et à la lincomycine (70 à 80\%). Nos résultats sont similaires à ceux de [8] au Burkina Faso qui a obtenu une sensibilité à la ciprofloxacine (95,5\%), gentamicine (87,5\%), Erythromycine $(86 \%)$ et une faible sensibilité à l'ampicilline $(33,3 \%)$. Cela pourrait s'expliquer par l'utilisation abusive des antibiotiques dans nos pays (Tableau IV).

Les aspects épidémiologiques et bactériologiques des infections urinaires chez la femme enceinte ont été abordés par plusieurs auteurs dans plusieurs pays africains et leurs résultats ont montré aussi des souches résistantes et multirésistantes [21-23]. Ces différents travaux montrent une fois encore la vulnérabilité des femmes enceintes, donc méritent que les médecins leur accordent plus de temps afin de pouvoir les aider à prévenir ces types d'infections et aussi à éviter l'automédication, responsable des résistantes émergentes ces dernières années.

Sensibilité de Candida albicans aux antifongiques testés. Les données de l'antifongigramme montrent une bonne sensibilité de Candida albicans aux antifongiques testés (Tableau V). Cette sensibilité est de : 80\% à la Miconazole ; Kétonazole; Econazole et à la Clotrinazole, et de $50 \%$ à la Fluconazole. Par contre, ce genre reste résistant à l'Amphotéricine $(93,33 \%)$ et à la Nystatine $(53,33 \%)$.

Tableau V: Profil de sensibilité globale aux antifongiques

\begin{tabular}{|c|c|c|c|c|c|c|c|}
\hline \multirow[t]{2}{*}{ Germe } & \multicolumn{6}{|c|}{ Nombre $(\%)$ de souches sensibles aux antifongiques } & \\
\hline & Miconazole & Kétonazole & Nystatine & Econazole & Clotrinazole & Fluconazole & Amphotéricine \\
\hline $\begin{array}{l}\text { Candida } \\
\text { Albicans }\end{array}$ & $15(80 \%)$ & $15(80 \%)$ & - & $15(80 \%)$ & $15(80 \%)$ & $8(50 \%)$ & - \\
\hline
\end{tabular}

- : Souches résistantes

Toutes les valeurs trouvées sont significativement différentes car $\mathrm{p}<0,05$.

\section{CONCLUSION}

L'infection urinaire chez la femme enceinte est relativement fréquente au CHUSS. L'infection touche toutes les tranches d'âges étudiés, on la retrouve aussi le plus souvent, au cours du deuxième et troisième trimestre de la grossesse. 
Les Bacilles à Gram Négatif étaient les germes les plus isolés au cours de l'infection urinaire chez la femme enceinte $(43,1 \%)$. Nous avons noté une forte résistance: Amoxicilline, amoxicilline+ acide clavulanique, céfoxitine et à la lincomycine. Par contre, ces mêmes bactéries ont une bonne sensibilité aux céftriaxone, ciprofloxacine, gentamicine, oxacilline, céphalexin et à l'Erythromycine.

\section{DECLARATION DE CONFLIT D'INTERET}

Les auteurs ne déclarent aucun conflit d'intérêt.

\section{REMERCIEMENTS}

Cette étude à bénéficié d'un financement du Fonds d'Appui pour la Recherche en Santé du Ministère de la Santé du Burkina Faso à travers un contrat de recherche (FARES 1/2013) accordé à Ibrahim SANGARE.

Nous tenons à remercier l'ensemble du personnel des centres de santé ayant participé à l'étude, le personnel du Département des laboratoires du CHUSS et particulièrement les femmes enceintes ayant participé à l'étude.

\section{REFERENCES}

[1] Thoulon J. M., Puech F. Boog G.Infections bactériennes et grossesse. Ellipses, Paris, 1995, 189-985.

[2] Legrain M., Durand D., Suc J. M., Jacob C. I. Tonthat H., Abrégé de néphrologie: Infection urinaire(Eds), Masson, Paris 1985, 39.

[3] Gelle P., Crepin G., Decoq J. Sulman C. H., Pyélites et infections urinaires de la gravido-puerperélité : Leur surveillance au moyen du néphrogramme isotonique. J. Gyn. Obst. Biol. Reprod. , 1972, 1), 676-690.

[4] Aghayan M., Thoumsin H. Lambotter A., Stratégies thérapeutiques de la bactériurie gravidique. Rev Med de Liège, 1990, 45(9), 433-439.

[5] Benomar L., Infections urinaires au cours de la grossesse. Université Hassan II, Casablanca, 2003, 200pages.

[6] Gassman V., Intérêt du dépistage urinaire en pratique gynéco obstétricale au CHU de 
Dakar. Med, 1990, 35(1), 1-9.

[7] Idatte J. M. Infections urinaires de l'adulte. G. Rechet Ed Ellipses, Paris, 1988, 207-240.

[8] Nikiéma A., Aspects épidémiologiques et bactériologiques des infections urinaires chez la femme enceinte. Université de Ouagadougou, Ouagadougou, 2002, 3-24pages.

[9] Olsen B. E., Hinderaker S. G., Lie R. T., Gasheka P., Baerheim A., Bergsjø P. KVÅLE G., The diagnosis of urinary tract infections among pregnant women in rural Tanzania; prevalences and correspondence between different diagnostic methods. Acta obstetricia et gynecologica Scandinavica, 2000, 79(9), 729-736.

[10] Olusanya O., Ogunledun A. Fakoya T., Asymptomatic significant bacteriuria among pregnant and non-pregnant women in Sagamu, Nigeria. West African journal of medicine, 1992, 12(1), 27-33.

[11] Coulibaly D., Infections urinaires et grossesse dans le centre de santé de référence du district de Bamako. Université de Bamako, Bamako, 2007, 81 pages.

[12] Diassana H. K., Infection urinaire et grossesse à la maternité Renée Cissé de Hamdallaye. Université de Bamako, Bamako, 2002, 151pages.

[13] Touré A., Infection urinaire et grossesse au centre de santé de référence de la commune II (CSREFCII). Université de Bamako, Bamako, 2012, 68pages.

[14] Sangare A., Association infection urinaire et grossesse dans le service de gynéco-obstétrique du centre hospitalo-universitaire Gabriel Touré : A propos de 106 cas. Université de Bamako, Bamako, 2009, 151pages.

[15] Koné H., Association infection urinaire et grossesse à la maternité de Hamdallaye (Centre de santé de référence de la commune IV) à propos de 35 cas. Bénin, 2002, 80 pages.

[16] Marrakchi O., Khrouf M., Ben Rejeb S., Ben Salah N., Chelli M. Boujnah A., La bactériurie asymptomatique chez la femme enceinte dans l'agglomération de Tunis: étude prospective. Tunisie médicale, 1986, 64(3), 217-220.

[17] Gebre S. S., Asymptomatic bacteriura in pregnancy: epidemiological, clinical, and microbiological approach. Ethiop Méd J, 1998, 36), 185-192.

[18] Combary A., Aspects épidémiologiques et bactériémiques des IU dans le service de pédiatrie du CHUYO. Université de Ouagadougou, Ouagadougou, 2000, 85pages. 
[19] Seydi M., Diouf A., Savadogo M., Sow A., Diop S. A., Soumare M., Diop B. M. Sow P. S., Infections du tractus urinaire au cours du sida a Dakar. Medecine Tropicale, 2009, 69(4), 406.

[20] Ouédraogo A. S., Somé D., Dakouré P., Sanon B., Birba E. Poda G., Profil bactériologique des infections du site opératoire au centre hospitalier universitaire Souro Sanou de Bobo Dioulasso. Laboratoire de Bactériologie-virologie, CHU Souro Sanou, Bobo Dioulasso, Burkina Faso, 2011, 19-39.

[21] Larabi K., Masmoudi A. Fendri C., Étude bactériologique et phénotypes de résistance des germes responsables d'infections urinaires dans un CHU de Tunis : à propos de 1930 cas. Médecine et maladies infectieuses 2003, 33), 348-352.

[22] Haouar I., Les infections urinaires à l'hôpital militaire d'instruction Mohammed V de Rabat : Fréquence, répartition et antibiorésistance des bactéries isolées dans les urines. Université Mohammed V, Rabat, 2010, 117pages.

[23] Boukadida J., Boukadida N. Elraii S., Profil et sensibilité aux antibiotiques de 2063 b a c t é r i e s uropathogènes isolées dans le centre de la Tu n is i e. Bull Soc Pathol Exot, 2002, 95(1), 8-10 


\section{INFECTION URINAIRE CHEZ LA FEMME ENCEINTE : ASPECTS EPIDEMIOLOGIQUES ET BACTERIOLOGIQUES A BOBO-DIOULASSO}

\section{RESUME}

Les infections urinaires sont les premières infections chez les femmes enceintes et peuvent entrainer des complications graves au cours de la grossesse. Afin de contribuer à l'amélioration de sa prise en charge, nous avons mené une étude transversale prospective, en vue de décrire ses aspects épidémiologiques et bactériologiques chez la femme enceinte au Centre Hospitalier Universitaire Souro Sanou (CHUSS). Sur le plan épidémiologique, la fréquence de l'infection urinaire était de $27,5 \%$ et touchait en majorité les femmes enceintes de 21 à 30 ans. E. coli, Staphylococcus aureus, Staphylococcus epidermis, Serratia, Acinetobacter, Enterobacter et Candida sont les germes isolés. Tous les germes sont très sensibles aux céphalosporines $(\mathrm{C} 3 \mathrm{G})$, aux aminosides, à l'oxacilline et à l'Erythromycine. Ces antibiotiques peuvent être prescrits aux femmes enceintes en attendant les résultats de l'examen cytobactériologique.

Mots clés : Antibiotique, CHUSS de Bobo-Dioulasso, E. coli, Examen cytobactériologique, Femme enceinte, Infection urinaire.

\section{How to cite this article:}

Taale E., Sanou S., Sangare I., Abdelkerim A. D., Mbatna A., Sirima C. and Savadogo A. Urinary tract infection among pregnant women at Bobo-Dioulasso: Epidemiological and bacteriological aspects. J. Fundam. Appl. Sci., 2016, 8(3), 1132-1145. 